\title{
A Coupled Model for the Emergence of Active Region Magnetic Flux into the Solar Corona
}

\author{
W. P. Abbett ${ }^{1,2}$, and G. H. Fisher ${ }^{1,2}$ \\ ${ }^{1}$ Space Sciences Laboratory, University of California, Berkeley, CA 94720-7450 \\ ${ }^{2}$ Institute for Theoretical Physics, University of California, Santa Barbara, CA 93106
}

\begin{abstract}
We present a set of numerical simulations that model the emergence of active region magnetic flux into an initially field-free model corona. We simulate the buoyant rise of twisted magnetic flux tubes initially positioned near the base of a stable, stratified model convection zone, and use the results of these calculations to drive a 3-D MHD model corona. The simulations show that time-dependent sub-surface flows are an important component of the dynamic evolution and subsequent morphology of an emerging magnetic structure. During the initial stages of the flux emergence process, the overlying magnetic field differs significantly from a force-free state. However, as the runs progress, and boundary flows adjust, most of the coronal field - with the exception of those structures located relatively close to the model photosphere - relaxes to a more force-free configuration. Potential field extrapolations do not adequately represent the magnetic structure when emerging active region fields are twisted. In the dynamic models, if arched flux ropes emerge with non-zero helicity, the overlying field readily forms sigmoid-shaped structures. However, the chirality of the sigmoid, and other details of its structure, depends on the observer's vantage point and the location within a given loop of emitting plasma. Thus, sigmoids may be an unreliable signature of the sign and magnitude of magnetic twist.
\end{abstract}

Subject headings: methods: numerical — MHD, Sun: corona, Sun: magnetic fields

\section{Introduction}

Active regions are areas of strong, bipolar magnetic field that represent the largest observable concentrations of magnetic flux on the Sun. Most important measures of solar activity, including solar flares, UV and X-ray radiation, and many (though not all) coronal 
This is an unedited preprint of an article accepted for publication in Astrophysical Journal. The final published article may differ from this preprint.

Copyright 2002 by The American Astronomical Society. Please cite as 'ApJ preprint doi:10.1086/344613'.

mass ejections (CMEs), are associated with active regions. Global properties of active regions are governed, at least in part, by photospheric and sub-surface magnetic fields and flows, and some of the most intense episodes of activity may well be related to the dynamic process of magnetic flux emergence from the high- $\beta$ convection zone into the low- $\beta$ environment of the solar corona.

Recently, a great deal of progress has been made in understanding the connection between active region magnetic field observed at the photosphere to magnetic field deep in the solar interior. A standard picture of active region formation and sub-surface evolution has emerged. In this picture, the bulk of the sub-surface magnetic field is stored well below the turbulent surface in a toroidal layer near the tachocline where it is thought to be generated (Gilman, Morrow, \& DeLuca 1989; DeLuca \& Gilman 1991; Parker 1993; MacGregor \& Charbonneau 1997; Durney 1997; Dikpati \& Charbonneau 1999). The tachocline (the narrow boundary that separates the radiative zone, which rotates as a solid body, and the convection zone, which exhibits latitudinal differential rotation) is located at or near the convective overshoot layer, and the toroidal field is subject to a variety of instabilities that can lead to the formation of buoyant magnetic flux ropes (Caligari et al. 1995; Fan \& Fisher 1996; Fan 2001; Wissink et al. 2000b; Brummell et al. 2002). These ropes then rise through the interior, interact with convection, and develop into twisted, fragmented $\Omega$-shaped structures that eventually emerge through the solar surface to form bipolar active regions (Dorch \& Nordlund 1998; Dorch et al. 1999; Abbett et al. 2000, 2001).

However, the detailed physics of the emergence of these magnetic structures through the many pressure scale heights of the solar photosphere, chromosphere, transition region, and low corona is less well understood, yet may be a critically important factor in the formation of filaments and prominences, and may contain clues to the triggering mechanism behind explosive and eruptive events. CMEs, for example, are often observed to be associated with newly emerging photospheric flux within pre-existing active regions (eg. Feynman \& Martin 1995; Feynman 1997). Since virtually every theoretical model of an eruptive event prescribes a particular magnetic field configuration and evolution at the photosphere (e.g. Antiochos et al. 1999a; Amari et al. 2000; Forbes 2000), a better understanding of how sub-surface conditions prior to and during the emergence of magnetic flux affect the solar corona (both globally and locally) should help further refine and improve existing models of CME initiation, and may also help to characterize the complex photospheric field signatures associated with these events.

To date, a complete, realistic dynamic simulation of the solar atmosphere from the base of the convection zone to the low corona remains computationally prohibitive. However, there are several useful approaches one can take when attempting to numerically model the process 
This is an unedited preprint of an article accepted for publication in Astrophysical Journal. The final published article may differ from this preprint.

Copyright 2002 by The American Astronomical Society. Please cite as 'ApJ preprint doi:10.1086/344613'.

of flux emergence through the surface layers. First, it is possible to perform detailed 3-D, fully compressible MHD calculations of the atmosphere, treating the energetics in the most realistic manner possible for the problem at hand (see Bercik 2002; Gudiksen \& Nordlund 2002). Although these types of calculations are generally computationally expensive, they have the advantage of being better suited for direct comparison with observational data, and could be used to simulate flux emergence at small spatial scales. Second, one can simplify the energy equation, and perform 3-D ideal MHD simulations of flux emergence at larger, active region size spatial scales (Fan 2001; Magara \& Longcope 2001). Simulations of this type self-consistently include the highly stratified surface layers in the computational domain, but to expedite the calculation, deeper layers are excluded.

In this paper, we present a third approach. We address the problem of flux emergence by creating a simple interface between two 3-D MHD codes: ANMHD (Fan et al. 1999), a code designed to efficiently model the low acoustic Mach number plasma of the solar convection zone, and a modified version of ZEUS-3D (Clarke et al. 1994; Stone \& Norman 1992a,b), a fully-compressible 3-D MHD code that we use to model the solar atmosphere from the photosphere to the low corona. This approach has the advantage of incorporating information from a sub-surface calculation into a simulation of the corona. The remainder of the paper is organized as follows: in Section 2, we describe the numerical methodology employed in both codes, and discuss the implementation of the code interface; in Section 3 we characterize our results, determine where the coronal magnetic field is most force-free in the simulations, and compare our dynamic simulations with a simple potential field extrapolation calculated using only the vertical component of magnetic field at the photospheric interface. Finally, in Section 4 we discuss the pros and cons of our approach, and in Section 5 we summarize our conclusions.

\section{Method}

The high- $\beta$ convection zone can be effectively modeled by numerically solving the 3D MHD equations in the anelastic approximation (Ogura \& Phillips 1962; Gough 1969; Glatzmeier 1984). This approximation results from a scaled variable expansion of the equations of compressible MHD about a zeroth order stratified reference state (see Lantz \& Fan 1999, and references therein). The time derivative of the perturbed density in the continuity equation is of high order in the expansion and is neglected. This results in significant computational savings, since the timesteps of the calculation are not restricted by rapidly propagating acoustic waves. This method is well suited to the study of turbulent convection (Lantz \& Fan 1999; Toomre 2000), and the physics of buoyant magnetic flux ropes deep in 
This is an unedited preprint of an article accepted for publication in Astrophysical Journal. The final published article may differ from this preprint.

Copyright 2002 by The American Astronomical Society. Please cite as 'ApJ preprint doi:10.1086/344613'.

the convection zone (Abbett et al. 2000; Fan 2001; Abbett et al. 2001); however, it is less suited for the detailed study of flux emergence through the photosphere, where $\beta$ approaches unity, and the acoustic Mach number is no longer small. In this realm, a fully-compressible treatment, such as that employed in ZEUS-3D, is required.

\subsection{The ANMHD Interior}

We take advantage of the efficiency of the anelastic method in our study of emerging flux by using the code ANMHD to first model the rise of an $\Omega$-loop through a stratified model convection zone. The following system of equations are solved numerically:

$$
\begin{gathered}
\nabla \cdot\left(\rho_{0} \mathbf{v}\right)=0 \\
\rho_{0}\left(\frac{\partial \mathbf{v}}{\partial t}+(\mathbf{v} \cdot \nabla) \mathbf{v}\right)=-\nabla p_{1}+\rho_{1} \mathbf{g}+\frac{1}{4 \pi}(\nabla \times \mathbf{B}) \times \mathbf{B}+\nabla \cdot \mathbf{\Pi} \\
\rho_{0} T_{0}\left(\frac{\partial s_{1}}{\partial t}+(\mathbf{v} \cdot \nabla)\left(s_{0}+s_{1}\right)\right)=\nabla \cdot\left(K \rho_{0} T_{0} \nabla s_{1}\right)+\frac{\eta}{4 \pi}|\nabla \times \mathbf{B}|^{2}+(\mathbf{\Pi} \cdot \nabla) \cdot \mathbf{v} \\
\frac{\partial \mathbf{B}}{\partial t}=\nabla \times(\mathbf{v} \times \mathbf{B})-\nabla \times(\eta \nabla \times \mathbf{B}) \\
\frac{\rho_{1}}{\rho_{0}}=\frac{p_{1}}{p_{0}}-\frac{T_{1}}{T_{0}} \\
\frac{s_{1}}{c_{p}}=\frac{T_{1}}{T_{0}}-\frac{\gamma-1}{\gamma} \frac{p_{1}}{p_{0}} .
\end{gathered}
$$

Here, $\rho_{1}, p_{1}, T_{1}, s_{1}, \mathbf{v}$, and $\mathbf{B}$ refer to the density, gas pressure, temperature, entropy, velocity, and magnetic field perturbations, and $\rho_{0}, p_{0}, T_{0}$, and $s_{0}$ denote the corresponding values of the zero-th order reference state, taken to be a field-free, adiabatically stratified polytrope of index $m=1.5 . \mathbf{g}=-\mathrm{g} \hat{\boldsymbol{z}}$ is the gravitational acceleration (assumed to be uniform), and $c_{p}$ denotes the specific heat at constant pressure. The viscous stress tensor $\boldsymbol{\Pi}$ is given by

$$
\Pi_{i j} \equiv \mu\left(\frac{\partial v_{i}}{\partial x_{j}}+\frac{\partial v_{j}}{\partial x_{i}}-\frac{2}{3}(\nabla \cdot \mathbf{v}) \delta_{i j}\right)
$$

where $\mu, \eta, K$, are the coefficients of viscosity, magnetic diffusion, and thermal diffusion respectively. The non-dimensional form of the above system of equations is solved in a Cartesian domain by spectral decomposition in the horizontal directions, and fourth-order centered differencing in the vertical direction. Horizontal boundaries are periodic, and the upper and lower boundaries are non-penetrating and stress-free. A complete description of 
This is an unedited preprint of an article accepted for publication in Astrophysical Journal. The final published article may differ from this preprint.

Copyright 2002 by The American Astronomical Society. Please cite as 'ApJ preprint doi:10.1086/344613'.

the numerical methodology employed by ANMHD can be found in Appendix A of Fan et al. (1999).

The simulations begin with a horizontal, buoyant magnetic flux tube positioned deep in the model convection zone. The tube is given a small entropy perturbation such that it evolves into an $\Omega$-shaped loop whose apex approaches the upper sub-photospheric boundary. We then use the time-dependent scalar variables and vector fields generated by ANMHD near this boundary to drive a simple ZEUS-3D simulation in Cartesian geometry of the photosphere, transition region, and lower corona. The two codes are thus coupled, though we have not allowed the low density, rapidly evolving corona to influence plasma below the photosphere. ANMHD simply provides a lower inflow boundary for the ZEUS-3D corona. We note that it is possible for the dynamic evolution of the corona to affect the MHD solution below the photosphere (see eg. Longcope \& Welsch 2000).

\subsection{The ZEUS-3D Atmosphere}

ZEUS-3D is a publicly available code that uses Eulerian, staggered finite differencing on a non-uniform mesh to numerically solve the following equations:

$$
\begin{gathered}
\frac{\partial \rho}{\partial t}+\nabla \cdot(\rho \mathbf{v})=0 \\
\frac{\partial \rho \mathbf{v}}{\partial t}+\nabla \cdot(\rho \mathbf{v} \mathbf{v})=-\nabla p-\rho g+\frac{1}{4 \pi}(\nabla \times \mathbf{B}) \times \mathbf{B} \\
\frac{\partial e}{\partial t}+\nabla \cdot(e \mathbf{v})=-p \nabla \cdot \mathbf{v}+Q \\
\frac{\partial \mathbf{B}}{\partial t}=\nabla \times(\mathbf{v} \times \mathbf{B})
\end{gathered}
$$

In the above, $\rho, p, \mathbf{v}$, and $\mathbf{B}$ have the their usual definitions of density, pressure, velocity, and magnetic field. The gravitational acceleration, $g$ is again assumed constant, and $e$ denotes the internal energy per unit volume (a $\gamma=5 / 3$ ideal gas is assumed). The interpolation is upstream and monotonic, and the internal energy and momenta are evolved using the Consistent Advection scheme introduced in (Norman et al. 1980). The Constrained Trans-

port algorithm (Evans \& Hawley 1988), modified with the Method of Characteristics (see Stone \& Norman 1992b), is used to evolve the magnetic field, which ensures that it remains divergence-free. An additional numerical viscosity term is added to the momentum equation to ensure stability.

Some simple modifications to ZEUS-3D were necessary to make the combined model feasible. First, to mimic in a simple way the heating and cooling necessary to maintain 
This is an unedited preprint of an article accepted for publication in Astrophysical Journal. The final published article may differ from this preprint.

Copyright 2002 by The American Astronomical Society. Please cite as 'ApJ preprint doi:10.1086/344613'.

the average temperature stratification seen in the solar atmosphere, we add an additional source term to the internal energy equation of the form $Q=k_{0}\left(T-T_{0}(z)\right)-k_{1} \rho^{2}$. In the first term, $T$ represents the temperature of the plasma, $T_{0}(z)$ is a specified, depthdependent temperature profile, and $k_{0}$ is an associated proportionality constant that scales as the inverse of a chosen heating (or cooling) time scale. In these early simulations, we are not attempting to realistically model the thermal structure of the solar atmosphere (as is done in eg. Lionello et al. 2001). Instead, the first part of our source term provides us with the ability to artificially heat or cool the atmosphere as necessary in order to maintain a particular temperature profile on a given timescale. The second term involves the square of the gas density $(\rho)$, and is included to approximate the effects of optically thin radiative cooling in cases where high density material entrained in emerging magnetic flux erupts into the low corona. Its associated proportionality constant, $k_{1}$, roughly scales as the inverse of the radiative cooling timescale. Second, we adjust the photospheric inflow boundary condition in ZEUS-3D so that the slopes of the initial density and internal energy profiles are maintained through the lower boundary zones.

We generate an equilibrium starting atmosphere; one whose lower boundary is anchored in the photosphere. $T_{0}(z)$ is specified so that a $1000 \mathrm{~km}$ thick combined model photosphere and chromosphere is kept at $5000 \mathrm{~K}$ (henceforth, this combined layer will be referred to as simply "the photosphere"). The corona is heated to $10^{6} \mathrm{~K}$, and in equilibrium, a geometrically thin transition layer is maintained. We must resolve the photospheric layers in the direction of the gravitational acceleration, where the pressure scale height is of order 100 $\mathrm{km}$. To achieve this, we first specify a non-uniform (but non-adaptive) vertical grid, where zones are highly concentrated in the photosphere and transition region (where the pressure drops by approximately 6 orders of magnitude), and are more sparsely allocated throughout the upper corona. We then assign a total mass to the domain, and allow the atmosphere to relax to a state of hydrostatic equilibrium. The ambient gas pressure in the photospheric boundary zones of the initial atmosphere is of sufficient strength to balance the magnetic pressure of the model active region introduced at the lower boundary (with a choice of initial axial field strength of $2.5 \times 10^{4} \mathrm{G}$ for a tube positioned at the base of the ANMHD domain); that is, the ratio of the gas pressure to the magnetic pressure $(\beta)$ near the top of the emerging loop is initially of order unity. Thus, we are able to simulate newly emerging magnetic flux as it expands through a $\beta \sim 1$ photospheric plasma into a low-density, low- $\beta$ model corona. The Cartesian box spans $75 \mathrm{Mm}$ on each side so that it is possible to model the initial stages of a modestly-sized emerging active region at realistic spatial scales, while still allowing the freedom to explore parameter space. 
This is an unedited preprint of an article accepted for publication in Astrophysical Journal. The final published article may differ from this preprint.

Copyright 2002 by The American Astronomical Society. Please cite as 'ApJ preprint doi:10.1086/344613'.

\subsection{The Interface Layer}

To generate a time-dependent inflow boundary for a ZEUS-3D simulation of the upper atmosphere, we first use ANMHD to simulate the sub-photospheric rise of initially horizontal, modestly twisted magnetic flux tubes positioned near the base of the model convection zone. We use a moderate resolution of $256 \times 128 \times 128$, where the longest dimension is along the axis of the initial tube, and the vertical dimension spans 5.1 pressure scale heights. Our initial field configuration has the form $\mathbf{B} \equiv B_{x}(r) \hat{\boldsymbol{x}}+B_{\theta}(r) \hat{\boldsymbol{\theta}}$. Here, the axial component of the field is given by $B_{x}(r) \equiv B_{0} e^{-r^{2} / a^{2}}$, where $r$ is the radial distance to the tube's central axis, $B_{0}$ is the initial axial field strength, and $a$ is the cross-sectional radius (taken to be a tenth the characteristic pressure scale height at the base of the model convection zone). The azimuthal component is given by $B_{\theta}(r) \equiv(q / a) r B_{x}(r)$, and the non-dimensional twist parameter $q$ is varied between runs. We run three separate simulations. In the first simulation (hereafter referred to S1), the twist parameter is set to zero, and thus the tube is initially untwisted. For the second simulation (dubbed S2), $q$ is taken to be 0.25 , which corresponds to $\sim 1.75$ field line rotations in the $280 \mathrm{Mm}$ horizontal extent of the simulation domain (note that the dimension of the ANMHD domain exceeds that of the ZEUS-3D domain, see Figure 1). We set $q$ to 0.5 in the third simulation (S3). Here, the initial tube is highly twisted, but not so much that it succumbs to the kink instability during its ascent. In each case, an initial entropy perturbation is applied to the central portion of the tube. This expedites the formation of a rising $\Omega$-shaped loop (see the discussion in Section 2 of Abbett et al. 2000).

We then identify a horizontal slice close to (but not at) the upper boundary of the ANMHD domain and save all the physical variables along this slice at successive time intervals. This provides us with a 2-D time-series for each run of the velocity, magnetic field, density, and internal energy perturbations that we use as a basis for the ZEUS-3D lower, driving boundary. Note that ANMHD provides magnetic field, velocity, density, and pressure perturbations about a polytropic reference state. The magnitude of these perturbations are given in terms of the axial magnetic field strength of the initial flux tube, and the gas density and pressure scale height at the base of the computational box. Thus, we have several degrees of freedom when specifying the values of the physical variables at the interface.

Since the anelastic approximation is generally unsuitable for simulating solar plasma at or very near the photosphere (note that the Alfvén speed, $c_{A}$, is the same order of magnitude as the sound speed, $c_{s}$, at the photospheric interface), we must assume that the gross properties of the field change only slightly in the narrow region between the sub-photospheric upper boundary of ANMHD and the photospheric lower boundary of ZEUS-3D. We assume $B_{0}$ (the field strength at the base of the model convection zone) is $2.5 \times 10^{4} \mathrm{G}$; this ensures that the maximum magnetic field strength at the interface boundary is of order $1000 \mathrm{G}$. The 
This is an unedited preprint of an article accepted for publication in Astrophysical Journal. The final published article may differ from this preprint.

Copyright 2002 by The American Astronomical Society. Please cite as 'ApJ preprint doi:10.1086/344613'.

unit of velocity at the interface layer is taken to be the local Alfvén speed at the photospheric boundary, $c_{A}$ (as opposed to the Alfvén speed of the sub-surface upper boundary provided by ANMHD), and the unit of time is self-consistently adjusted $\left([t]=[z] / c_{A}\right)$. This choice of scaling at the boundary leads to average flow speeds during the course of the coronal simulation that are higher (several times the local Alfvén Mach number) than the draining velocities observed in newly emerging arch filaments (see Soru-Escaut \& Mouradian 1990; Tsiropoula et al. 1992). However, this scaling is necessary to emerge flux into the initially field-free model corona at timescales where numerical diffusion does not become a dominant process. In practice, we find that the model corona responds rapidly to the slow evolution of the photospheric boundary, thus the increased velocities coupled with the shorter evolutionary timescale at the photospheric boundary allow us to minimize the unphysical effects of excessive numerical diffusion and more faithfully describe the dynamic processes inherent to the initial emergence. After flux fills the model corona (eg. after $t=9$ in run S2), the photospheric inflow velocities are cut by a factor of 4 to substantially sub-Alfvénic values, and the model corona is allowed to relax.

\section{Results}

Figure 1 is an illustration of how an ANMHD simulation of a rising $\Omega$-loop can drive a ZEUS-3D simulation of the solar corona. The red rectangular box in the lower portion of the figure outlines the 256x128x128 ANMHD simulation domain, and within the box is a volume rendering of the magnetic field strength, $|\mathbf{B}|$, for the second to last timestep of run S2. The white axes positioned above the ANMHD box trace the outer boundaries of a relatively low resolution $(64 \times 64 \times 64)$ ZEUS-3D simulation of flux emergence at a time late in the run when a significant amount of flux has already been introduced through the lower boundary into the initially field-free model atmosphere. The grayscale image overlying the boundary face is an "artificial magnetogram", and represents the vertical component of the magnetic field $B_{z}$ at the interface boundary. Dark areas in the image represent regions of negative polarity, and light areas represent regions of positive polarity. Magnetic field lines emanating from places at the boundary where $B_{z}$ is strong are shown in various shades of blue - the lighter the color, the stronger the vertical field at the photospheric footpoint. Near the top of ANMHD's rectangular domain is a dark gray, horizontal slice though the volume. Vector fields and density perturbations along this plane are used to specify the physical variables at the lower boundary of ZEUS-3D at the timestep shown. The image appearing on the slice is a cut-away of $|\mathbf{B}|$ near the apex of the loop. In both codes, the grid spacing of each zone is $1 \mathrm{Mm}$ per side at the interface; thus, the emerging active region of Figure 1 is roughly $40 \mathrm{Mm}$ across. 
This is an unedited preprint of an article accepted for publication in Astrophysical Journal. The final published article may differ from this preprint.

Copyright 2002 by The American Astronomical Society. Please cite as 'ApJ preprint doi:10.1086/344613'.

As the $\Omega$-loop rises through the ANMHD convection zone, a characteristic flow field develops around the apex of the loop. In the case of run S2, the initial field line twist and relatively high degree of loop curvature prevent substantial fragmentation near the apex as it approaches the photospheric boundary. Still, the interaction of oppositely directed vortex pairs shed during the tube's ascent causes a noticeable lack of cohesion near the loop's apex. This loss of cohesion is most evident in run S1 where at the apex, the sub-surface loop cross-section resembles a thin sheath of axial field embedded in vertical flows, followed by concentrations of field that reside along the axes of the vortex pairs (see eg. the bottom frame of Figure 2 in Abbett et al. 2000). Thus, we do not simulate the emergence of a single, well-defined (highly twisted) horizontal flux rope positioned just below the photosphere (as was done by Magara \& Longcope 2001; Fan 2001), rather we emerge magnetic flux present at the top of a buoyant, weakly twisted, often fragmented $\Omega$-loop - though this configuration results from an initially horizontal tube positioned near the base of our model convection zone. Since the morphology of the magnetic field in the apex cross-section differs substantially between each of the ANMHD simulations S1-S3, we choose as a standard reference between runs the unit of time of each non-dimensional ANMHD calculation used to generate the driving boundaries: $[t]=[z] /[v]$. Here, $[z]=H_{r}$ and $[v]=B_{0} / \sqrt{4 \pi \rho_{r}}$ are the characteristic length scale and velocity of the sub-surface calculations, and are given in terms of $H_{r}$, $\rho_{r}$, and $B_{0}$ : the pressure scale height and density at the base of the computational domain, and the axial field strength along the initial tube.

Different stages of the emergence process are shown in Figure 2 for the case where ANMHD run S2 drives the ZEUS-3D corona $(t=8.25$ in the top row, and 9.00 in the bottom row). The left column shows the magnetic field at the interface layer (the gray-scale image corresponds to the vertical component of the field, and the vectors indicate the direction of the horizontal field along the interface); the right column shows the corresponding snapshot of the model corona. Fieldlines are traced with different shades of gray: the stronger the vertical magnetic field at the photospheric boundary, the lighter the shade. The presence and distribution of the vertical component of velocity at the interface is important in our simulations, since the bulk of the magnetic field at the loop apex is oriented in a direction parallel to the photospheric boundary. In this version of ZEUS-3D, magnetic diffusion is not explicitly included, only numerical diffusion due to truncation error is present. Thus, a vertical plasma flow through the interface boundary is necessary in order to transport magnetic field into our model corona while conserving flux. To illustrate this point, consider the following representation of the $z$-component of the ideal MHD induction equation:

$$
\frac{\partial B_{z}}{\partial t}+\nabla_{\perp} \cdot\left(\mathbf{v}_{\perp} B_{z}\right)=\nabla_{\perp} \cdot\left(\mathbf{B}_{\perp} v_{z}\right)
$$

Here, the subscript $\perp$ denotes directions perpendicular to the vertical direction, $z$. The 
This is an unedited preprint of an article accepted for publication in Astrophysical Journal. The final published article may differ from this preprint.

Copyright 2002 by The American Astronomical Society. Please cite as 'ApJ preprint doi:10.1086/344613'.

lefthand side of this equation shows that in the absence of a vertical velocity and a horizontal magnetic field below the surface, vertical signed flux is conserved. It is the righthand side of this equation that represents the sources and sinks of magnetic flux due to emergence or submergence.

The left column of Figure 3 shows how the local coronal magnetic field in our model has evolved in response to the initial dynamic emergence of three $\Omega$-loops of different helicity. The first row (left column) is a snapshot from a coronal simulation driven by ANMHD run S1 (where the initial non-dimensional twist parameter $q$ was set to zero), the middle row corresponds to the simulation driven by run S2 (where $q=0.25$ ), and the bottom row is a snapshot of the simulation driven by run S3 ( $q=0.5$, the maximum amount of initial twist). Magnetic field lines are shown in various shades of gray - again, the lighter the shade, the stronger the vertical field at the footpoint (for clarity, only the stronger fieldlines are shown). The computational domain is viewed from a vantage point above the top boundary (looking directly down on the photosphere), thus we see the projections of the field lines onto the surface. The grayscale images anchoring the fieldlines are artificial magnetograms, and display the vertical component of the magnetic field at each interface boundary. The right column of Figure 3 shows (for runs S1-S3) the fieldlines that result from a potential field calculation that uses only the snapshot of the vertical component of the magnetic field at each interface layer.

It is clear in the middle and bottom rows of Figure 3 that for the dynamically evolving twisted $\Omega$-loops, the potential field calculation differs substantially from the MHD solution. This is not surprising, since the potential field has no way of recreating the past history of the emerging structure. In the dynamic calculations, as the flux rope begins to expand into the corona, the fieldlines emerge in an orientation roughly parallel to a line drawn through the center of each magnetic polarity. This initial orientation is indicative of the magnitude and sign of the field line twist assigned to the buoyant horizontal flux tube initially positioned at the base of the ANMHD model convection zone. As time goes on, and the leading and trailing polarities separate, their tilt angle (the angle between the line drawn through the center of each magnetic polarity and the $\hat{x}$ direction) is reduced. Field lines become increasingly twisted, and "sigmoidal" structures appear in regions above the active region where the coronal field strength is high.

The potential extrapolation appears to fare much better with the initially untwisted tube pictured in the top row of Figure 3. However, the atmosphere is still in a very dynamic stage of the calculation, and the field (even toward the top of the domain) is not at all force-free. To illustrate this further, we calculate the angle $\theta=\cos ^{-1}|\mathbf{J} \cdot \mathbf{B} /(|\mathbf{J}||\mathbf{B}|)|$ along fieldlines from the same dynamic snapshots pictured in the bottom two rows (left column) of 
This is an unedited preprint of an article accepted for publication in Astrophysical Journal. The final published article may differ from this preprint.

Copyright 2002 by The American Astronomical Society. Please cite as 'ApJ preprint doi:10.1086/344613'.

Figure 3 and plot this result in Figure 4. The angle $\theta$ is a measure of how force-free the field is at a given location along a fieldline - if $\theta$ is zero, the current and magnetic field vectors are parallel, $\mathbf{J} \times \mathbf{B}=0$, and the field is force-free. Conversely, if $\theta=\pi / 2$, the current is perpendicular to the field, and the situation is highly dynamic. In Figure 4, we plot $\theta$ along individual fieldlines: bright blue corresponds to a $\theta$ of zero, and bright magenta indicates a value of $\pi / 2$. Black represents $\theta=\pi / 4$; thus, the more blue a fieldline, the more force-free the magnetic field is at that point.

The top left frame of Figure 5 is a more quantitative view of the value of $\theta$ along a slice $8000 \mathrm{~km}$ directly above the S2 model active region at $t=8.75$. Values of $\theta$ vary widely, from a minimum of 0.7 degrees in some of the strong-field regions above the bipolar region, to roughly 45 degrees in the high density region oriented above and along the photospheric neutral line, and up to as 85 degrees along the edges of the flux rope (and in regions of weaker fields and currents far afield from the emerging structure). This is a consequence of the imposed flow field at the boundary — mass is being introduced into the low corona along with magnetic field; thus dense structures (consistent with chromospheric densities) and strong localized flows quickly develop in the low corona over the active region. This is reflected in the second column of Figure 5, where the plasma $\beta$ and Alfvén Mach number $\left(M_{A}\right)$ along the surface are shown. $\beta$ and $M_{A}$ are relatively large in dynamic regions where the magnetic field is weak, or where the density is relatively high. Note that in these simulations, we are emerging flux into an initially field free region. Since the only means of transporting flux into the domain in an ideal calculation is via a vertical flow field across the interface boundary, it is not surprising that relatively large Alfvén Mach numbers that exceed the sound speed can be seen in regions near the photosphere where transverse velocities dominate, and only a small amount of field is present (eg. at and beyond the edges of the emerging structure). For reference, the lower left panel shows the vertical component of the magnetic field at the photospheric boundary.

Although the flow patterns, density, and orientation of the structure above the model active region is reminiscent of a small emerging arch filament, we note that our treatment of the energy equation is only approximate, and our imposed flow fields and emergence timescales (by necessity) are more rapid than is observed. Thus, we caution against an over-interpretation of the initial stages of our simulations. As the runs progress, projections of the magnetic fieldlines (at this height and above) exhibit a distinctive sigmoidal shape, particularly if one views the entirety of a given fieldline from the apex of the loop to its footpoints (see Figure 6). Though our simple computational models do not properly describe the detailed energetics of the solar corona, we are able to describe the basic structure and evolution of the field. We feel that it is reasonable to assume the presence of soft X-ray emitting material along the strong magnetic fieldlines of our model coronal loops, and in this 
This is an unedited preprint of an article accepted for publication in Astrophysical Journal. The final published article may differ from this preprint.

Copyright 2002 by The American Astronomical Society. Please cite as 'ApJ preprint doi:10.1086/344613'.

manner, we can use the projections obtained from our simulations (as shown in Figures 2, 3 and 6) as proxies for the location and shape of sigmoids that may be associated with emerging, twisted $\Omega$-loops.

However, it is not immediately apparent how to determine the chirality of the sigmoid associated with a particular model active region; that is, if the curve of the magnetic fieldline projections indicate whether emitting plasma will form a Z-shape or an S-shape when viewed from above. First, as can be seen in some of the stronger fieldlines of Figure 6, the chirality of a sigmoid-shaped projection often depends upon which loop or which portion of a given loop is viewed. For example, if the entire fieldline from footpoint to footpoint is considered, many of the projections of overlying fieldlines in Figures 3 and 6 form distinct, rounded Z-shapes. Conversely, if only the top of the loop is considered, the same fieldlines may instead exhibit no discernible sigmoid. Second, if fieldlines closer to the photosphere in Figure 6 contain the emitting plasma, an S-shape is evident. Though our initial flux rope positioned at the base of the ANMHD convection zone is only weakly twisted, and these morphological distinctions in the associated model corona are subtle, our results imply that the shape of a sigmoid can be a projection effect, and depends upon the location within a given magnetic structure of emitting plasma. We note that the temperature of plasma entrained along fieldlines may vary with height in the corona. It is likely that the high density material located close to the photosphere is a product of the eruption of material into the corona as an $\Omega$-loop begins to emerge. It is natural to assume that this material is initially cooler than X-ray emitting temperatures, and instead radiates at longer wavelengths. Then during the initial stages of our simulations, many of the sigmoid-shaped fieldline projections that are located near to the photosphere may be better proxies for filament-like structures, rather than X-ray sigmoids.

As vertical velocities at the model photosphere are reduced (eg. after $t=9$ in run $\mathrm{S} 2$ ), flux is no longer being rapidly driven into the corona, and the coronal field begins to relax to a more force-free configuration. This is shown in Figure 7 for a later stage of the model corona driven by run S3 $(t=9.25)$, where except for regions very close to the photosphere, values of $\theta$ are significantly less than those calculated during the initial stages of the simulations $(t \sim 8)$. The relatively narrow region close to the interface boundary in Figure 7 (where the fieldlines are dark to magenta colored) is a region where currents and fields are relatively strong, densities are high, and the field configuration is the least forcefree. We note that although regions of dynamic interest lie in a relatively narrow region close to the lower boundary, the grid is non-uniform and concentrated in these layers, thus the volume is sufficiently resolved. 
This is an unedited preprint of an article accepted for publication in Astrophysical Journal. The final published article may differ from this preprint.

Copyright 2002 by The American Astronomical Society. Please cite as 'ApJ preprint doi:10.1086/344613'.

\section{Discussion}

While our approach allows us to explore the consequences of driving a 3-D compressible MHD model corona with magnetic fields and flows generated by fully self-consistent, 3-D MHD simulations of twisted, buoyant $\Omega$-loops in the solar interior, we feel that it is important to point out some of the limitations of our technique. As with all explicit computational schemes, we are bound by the CFL condition; which in our case, puts rather severe restrictions on the size of the timestep (for example, densities in the coronal portion of our model are low, while magnetic field strengths in active regions can be quite high). Thus, it is impractical to perform large explorations of parameter space at spatial and temporal scales that approach the true size and evolutionary timescale of active region arcades and coronal loops on the Sun. For example, at realistic timescales, interesting physics that is simulated in early stages of a numerical calculation will succumb to the cumulative effects of numerical diffusion over time. Additionally, it is desirable to maintain the consistency of the vector fields and timescales prescribed by the code that drives the lower boundary. Arbitrary scaling of the velocity field at the interface without similarly adjusting the emergence rate can result in unphysical behavior in the model corona; since, for example, equation 13 may no longer be satisfied at the boundary. In our case, we scale the boundary velocities in the most self-consistent way possible (as described in Section 2.3), and increase the frequency of boundary updates such that equation 13 is satisfied at the interface. Thus, the calculation becomes feasible, but the initial flows are quite strong and the atmosphere is more dynamic than is likely to be the case on the Sun. However, since the model corona responds very rapidly to changes at the interface boundary, we feel that the general characteristics of these types of simulations - the dynamic nature of the flux emergence process (for example, how photospheric upflows force transverse field into pre-existing coronal loops or arcades) - are in many respects similar to the general properties of active region flux emergence on the Sun.

One could argue that it is more self-consistent to model emerging magnetic flux by following the dynamic evolution of an initially motionless, horizontal buoyant magnetic flux rope positioned just below the photosphere at the base of a self-contained model atmosphere (as was recently done by Fan 2001; Magara \& Longcope 2001), neglecting plasma flows and other information obtained by first simulating the rise of a loop through the entirety of a model convection zone. We choose not to take that approach here, since one of our future goals is to develop a modeling framework whereby high resolution vector magnetograms of active regions (and observations of flow fields in and about these regions) can be used to drive dynamic models of the solar corona. Since there is little sub-surface data available, computational models of the low corona must rely upon an observationally obtained lower boundary layer to drive the simulations. By using ANMHD to artificially generate this layer, 
This is an unedited preprint of an article accepted for publication in Astrophysical Journal. The final published article may differ from this preprint.

Copyright 2002 by The American Astronomical Society. Please cite as 'ApJ preprint doi:10.1086/344613'.

we hope to determine the feasibility and efficacy of this approach. In the near future, we will perform detailed comparisons of these simple code-coupling models with models that self-consistently treat the emergence of magnetic flux through photosphere, transition region, and corona to determine whether, for example, it is justifiable to neglect the effects of the coronal field on the sub-surface plasma.

\section{Conclusions}

We have presented a set of numerical experiments designed to simulate the flux emergence process by driving a 3-D MHD model corona with a self-consistent 3-D simulation of a twisted $\Omega$-loop that has risen buoyantly from the base of a stratified, stable model convection zone. We find that:

- The presence and distribution of boundary flows resulting from the 3-D sub-surface evolution of an $\Omega$-loop is important to the dynamic evolution and coronal morphology of an emerging structure. The component of the flow perpendicular to the boundary layer is particularly important to the dynamic emergence process, since (in an ideal MHD calculation) such a flow is necessary to transport magnetic field into the model corona while conserving flux.

- At least during the initial stages of the flux emergence process, the simulations suggest that the magnetic field in most regions surrounding the emerging structure differs substantially from a force-free configuration. As the $\Omega$-loop emerges, and the velocity and magnetic fields at the photosphere evolve, less flux is transported into the model corona, and with the exception of structures close to the dynamic photospheric boundary, the overlying field relaxes to a more force-free state.

- Potential field extrapolations calculated from the vertical component of the magnetic field at the photosphere do not accurately describe the detailed magnetic structure of the low corona overlying an emerging, twisted active region.

- Fieldlines generated from arched flux ropes that emerge with non-zero helicity will form sigmoid-shaped features when viewed from above. However, the chirality of a sigmoid, and other details of its structure depend on projection effects, viewing angle, and the location within a given magnetic loop of emitting plasma.

This work was supported by the DoD/AFOSR MURI grant "Understanding Magnetic Eruptions and their Interplanetary Consequences", NASA's SECT and Solar Physics Re- 
This is an unedited preprint of an article accepted for publication in Astrophysical Journal. The final published article may differ from this preprint.

Copyright 2002 by The American Astronomical Society. Please cite as 'ApJ preprint doi:10.1086/344613'.

search and Analysis programs, and by NSF. We wish to thank the Institute for Theoretical Physics at UCSB for their support through their program on Solar Magnetism, and for their hospitality during our stay in 2002. We also wish to thank the authors of the original ZEUS-3D package for making their code and documentation publicly available. 
This is an unedited preprint of an article accepted for publication in Astrophysical Journal. The final published article may differ from this preprint.

Copyright 2002 by The American Astronomical Society. Please cite as 'ApJ preprint doi:10.1086/344613'.

\section{REFERENCES}

Abbett, W. P., Fisher, G. H., \& Fan, Y., 2001, ApJ, 546, 1194.

Abbett, W. P., Fisher, G. H., \& Fan, Y., 2000, ApJ, 540, 548.

Amari, T., Luciani, J. F., Mikic, Z., and Linker, J., 2000, ApJL, 529, L49.

Antiochos, S. K., Devore, C. R., and Klimchuk, J. A., 1999, ApJ, 510, 485.

Bercik, D. J., 2002, PhD thesis, Michigan State University.

Brummell, N., Cline, K., \& Cattaneo, F., 2002, MNRAS, 329, L73.

Caligari, P., Moreno-Insertis, F., \& Schüssler, M., 1995, ApJ, 441, 886.

Clarke, D. A., Norman, M. L., \& Fiedler, R. A., 1994, "ZEUS-3D User Manual", Ver. 3.2.1, NCSA publication, Univ. of Illinois.

DeLuca, E. E., \& Gilman, P. A., 1991, in Solar Interior and Atmosphere, Tucson AZ, University of Arizona Press, 275.

Dikpati, M., \& Charbonneau, P., 1999, ApJ, 518, 508.

Dorch, S. B. F., \& Nordlund, A., 1998, A\&A, 338, 329.

Dorch, S. B. F., Archontis, V., \& Nordlund, A., 1999, A\&A, 352, L79.

Durney, B. R., 1997, ApJ, 486, 1065.

Fan, Y., 2001, ApJ, 554, L111.

Fan, Y., 2001, ApJ, 546, 509.

Fan, Y., Fisher, G. H., 1996, Sol. Phys., 166, 17.

Fan, Y., Zweibel, E. G., Linton, M. G., \& Fisher, G. H., 1999, ApJ, 521, 460.

Feynman, J., 1997, "Evolving Magnetic Structures and CMEs", in "Coronal Mass Ejections", ed. Crooker, N., Joselyn, J. A., and Feynman, J., Geophysical Monograph 99, AGU, 49.

Feynman, J., and Martin, S. F., 1995, J. Geophys. Res., 100, 3355.

Forbes, T. G., 2000, J. Geophys. Res., 105, 23153. 
This is an unedited preprint of an article accepted for publication in Astrophysical Journal. The final published article may differ from this preprint.

Copyright 2002 by The American Astronomical Society. Please cite as 'ApJ preprint doi:10.1086/344613'.

Gilman, P. A., Morrow, C. A., \& DeLuca, E. E., 1989, ApJ, 338, 528.

Glatzmeier, G. A., 1984, J. Comput. Phys., 55, 461.

Gough, D. O., 1969, J. Atmos. Sci., 26, 448.

Gudiksen, B. V., \& Nordlund, A., 2002, ApJ, 572, L113.

Evans, C. R., \& Hawley, J. F., 1988, ApJ, 33, 659.

Lantz, S. R., \& Fan, Y., 1999, ApJS, 121, 247.

Lionello, R., Linker, J. A., \& Mikic, Z., 2001, ApJ, 546, 542.

Longcope, D. W., \& Welsch, B. T., 2000, ApJ, 545, 1089.

MacGregor, K. B., \& Charbonneau, P., 1997, ApJ, 486, 484.

Magara, T., \& Longcope, D. W., 2001, ApJ, 559, L55.

Norman, M. L., Wilson, J. R., \& Barton, R. T., 1980, ApJ, 239, 968.

Ogura, Y., \& Phillips, N. A., 1962, J. Atmos. Sci., 19, 173.

Parker, E. N., 1993, ApJ, 408, 707.

Soru-Escaut, I., \& Mouradian, Z., 1990, A\&A, 230, 474.

Stone, J. M., and Norman, M. L., 1992, ApJS, 80, 753.

Stone, J. M., and Norman, M. L., 1992, ApJS, 80, 791.

Tsiropoula, G., Georgakilas, A. A., Alissandrakis, C. E., \& Mein, P., 1992, A\&A, 262, 587.

Toomre, J., 2000, "Turbulent Convection and Differential Rotation Within the Sun", in "Recent Insights into the Physics of the Sun and Heliosphere, Highlights from SOHO and other Space Missions", ASP Conf. Ser., 200.

Wissink, J. G., Proctor, M. R. E., Matthews, P. C., Hughes, D. W., 2000, MNRAS, 318, 501. 
This is an unedited preprint of an article accepted for publication in Astrophysical Journal. The final published article may differ from this preprint.

Copyright 2002 by The American Astronomical Society. Please cite as 'ApJ preprint doi:10.1086/344613'.

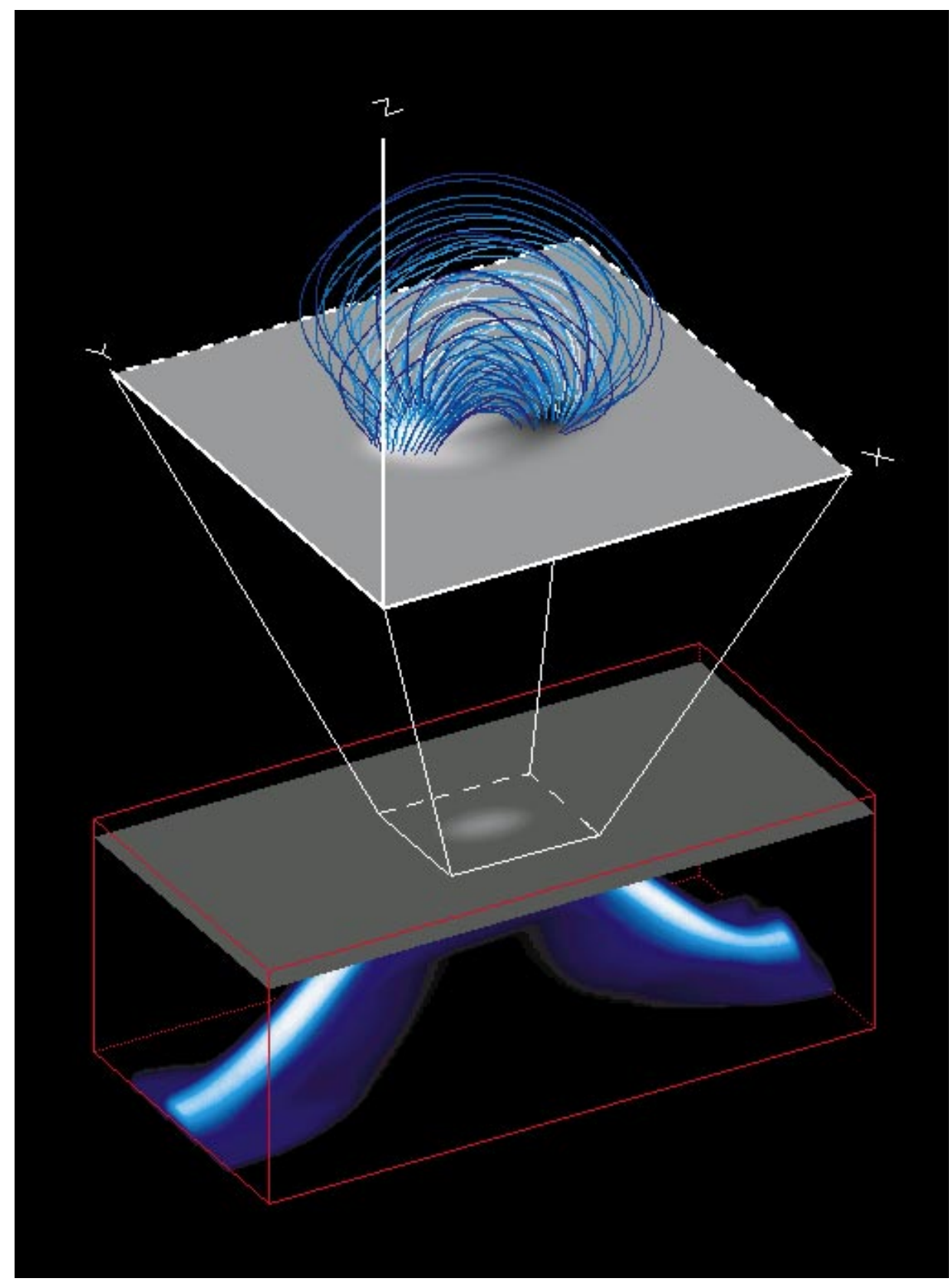

Fig. 1. - A snapshot of a ZEUS-3D simulation of the model corona being driven by an emerging $\Omega$-loop calculated by ANMHD. Within the ANMHD domain (the lower rectangular box) is a volume rendering of $|\mathbf{B}|$, and within the ZEUS-3D domain (the upper axes) some of the magnetic fieldlines are traced in blue. The vertical component of the magnetic field along the ZEUS-3D photospheric lower boundary is shown as a grayscale image: the light areas represent regions of positive polarity, and dark areas represent negative polarity. See text for details. 
This is an unedited preprint of an article accepted for publication in Astrophysical Journal. The final published article may differ from this preprint.

Copyright 2002 by The American Astronomical Society. Please cite as 'ApJ preprint doi:10.1086/344613'.

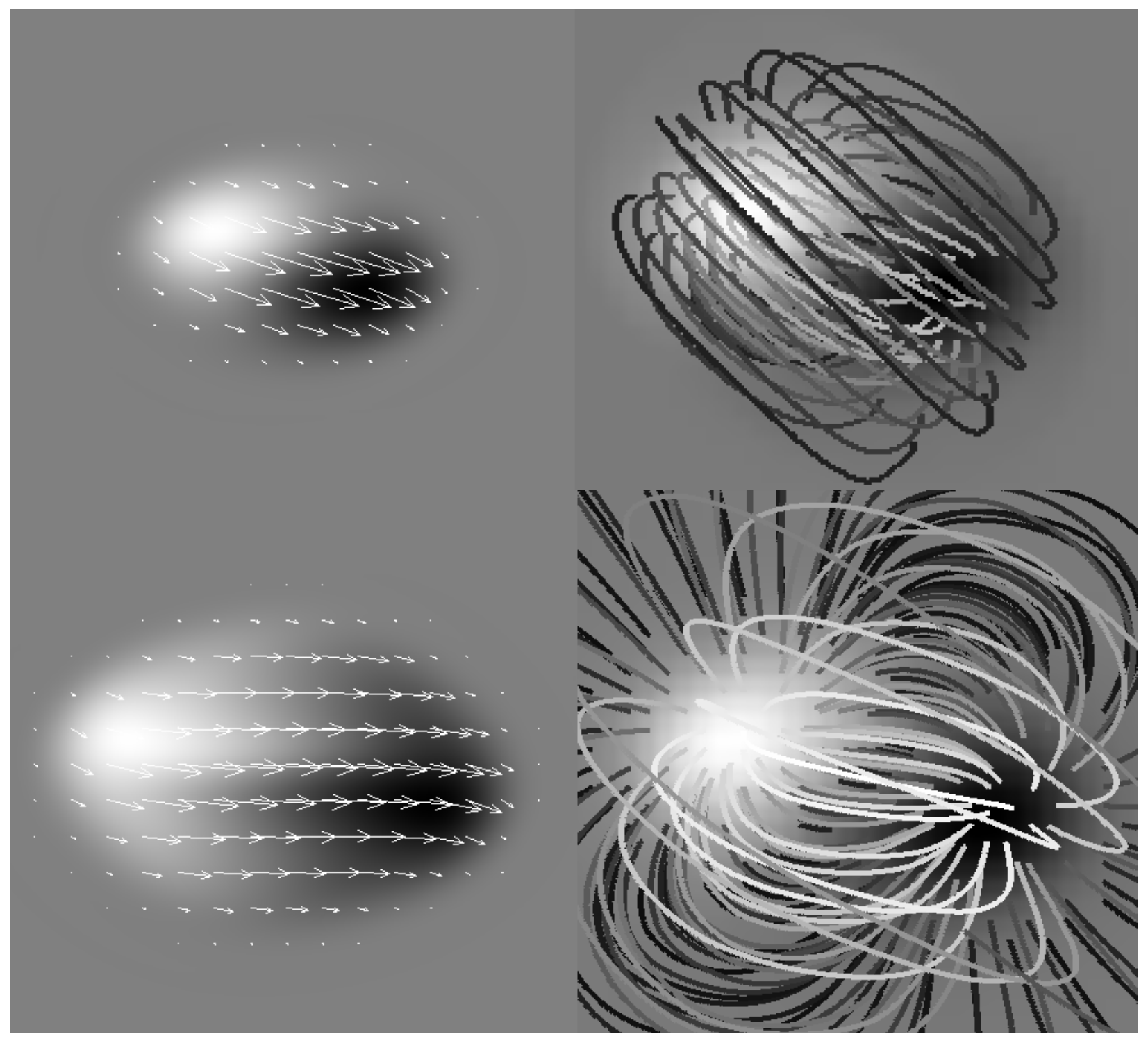

Fig. 2.- The Zeus-3D model corona being driven by ANMHD run S2 at two different stages of emergence ( $t=8.25$ in the top row and $t=9$ in the bottom row). The left column shows the vector magnetic field at the interface boundary (note that the grayscale image indicates vertical field strength, and the arrows indicate horizontal field strength and direction). The right column shows the corresponding model corona. Fieldlines are traced in gray: the lighter the shade, the stronger the vertical component of the field at the photospheric boundary. For clarity, not all fieldlines are shown. 
This is an unedited preprint of an article accepted for publication in Astrophysical Journal. The final published article may differ from this preprint.

Copyright 2002 by The American Astronomical Society. Please cite as 'ApJ preprint doi:10.1086/344613'.

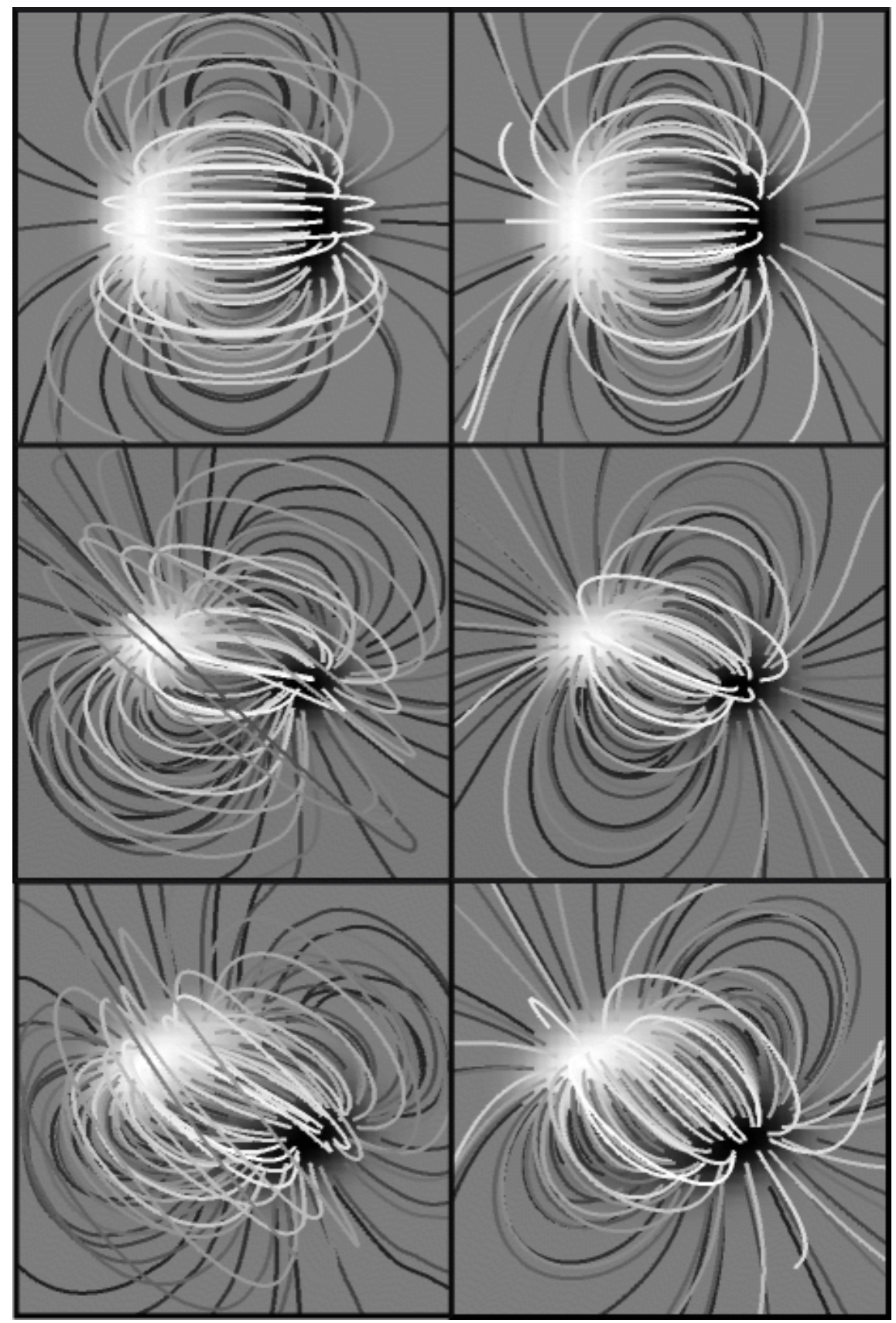

Fig. 3.- A qualitative comparison at $t \sim 9$ of three dynamic model coronae (left column) with corresponding potential field calculations (right column). The photospheric boundary of the first row is given by ANMHD run S1 (an initially untwisted flux rope), and the middle and bottom rows are given by runs $\mathrm{S} 2$ and $\mathrm{S} 3$ (initially twisted flux ropes) respectively. The lighter the shade, the stronger the field at the photospheric footpoint. 
This is an unedited preprint of an article accepted for publication in Astrophysical Journal. The final published article may differ from this preprint.

Copyright 2002 by The American Astronomical Society. Please cite as 'ApJ preprint doi:10.1086/344613'.

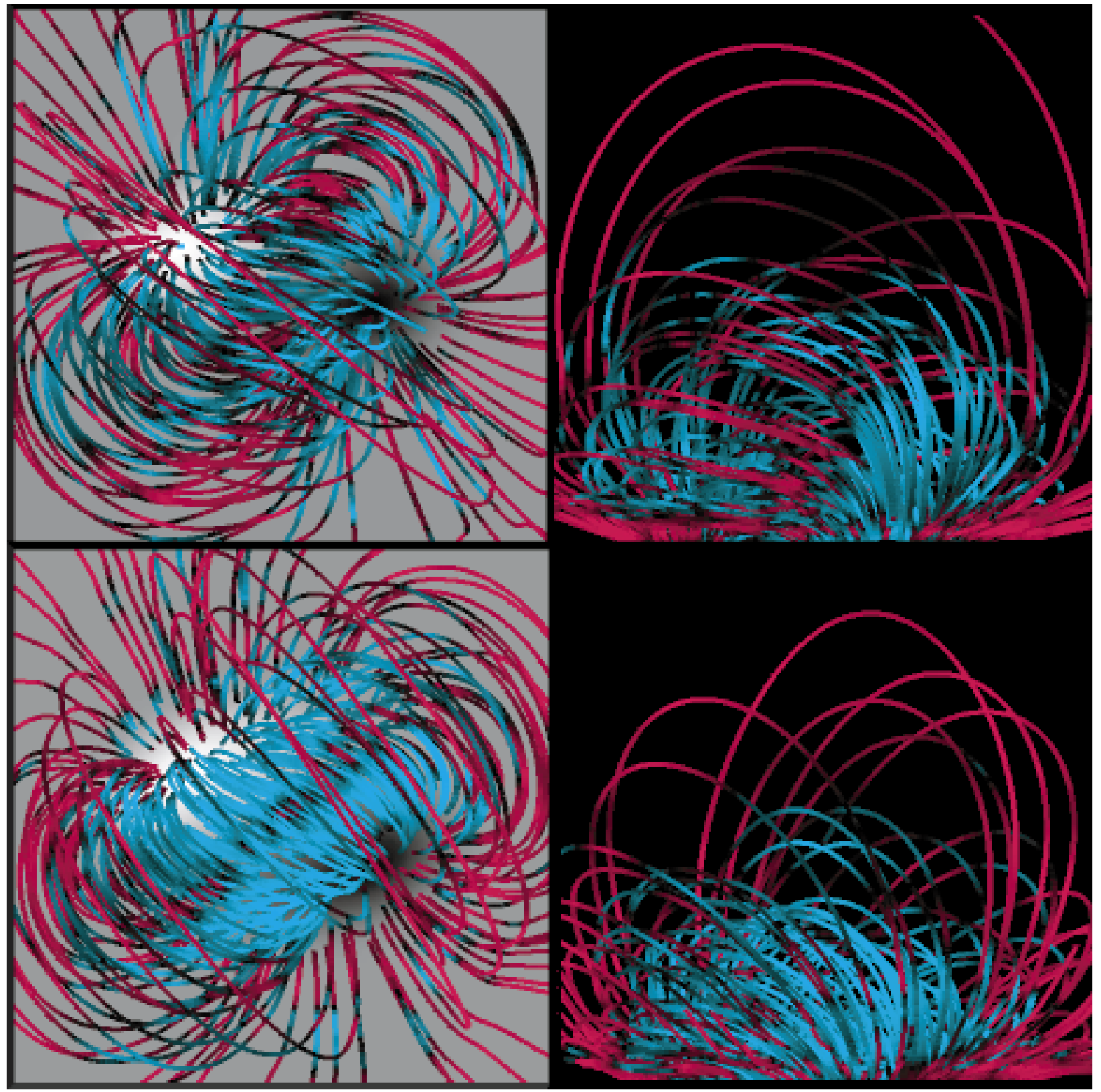

Fig. 4.- A qualitative measure of how force-free the field is along a number of fieldlines. The top row corresponds to the dynamic snapshot shown in the middle row (left column) of Figure 3, and the bottom row corresponds to the atmosphere shown in the bottom row (left column) of Figure 3. The more blue the color, the more force-free the field. See text for details. 
This is an unedited preprint of an article accepted for publication in Astrophysical Journal. The final published article may differ from this preprint.

Copyright 2002 by The American Astronomical Society. Please cite as 'ApJ preprint doi:10.1086/344613'.
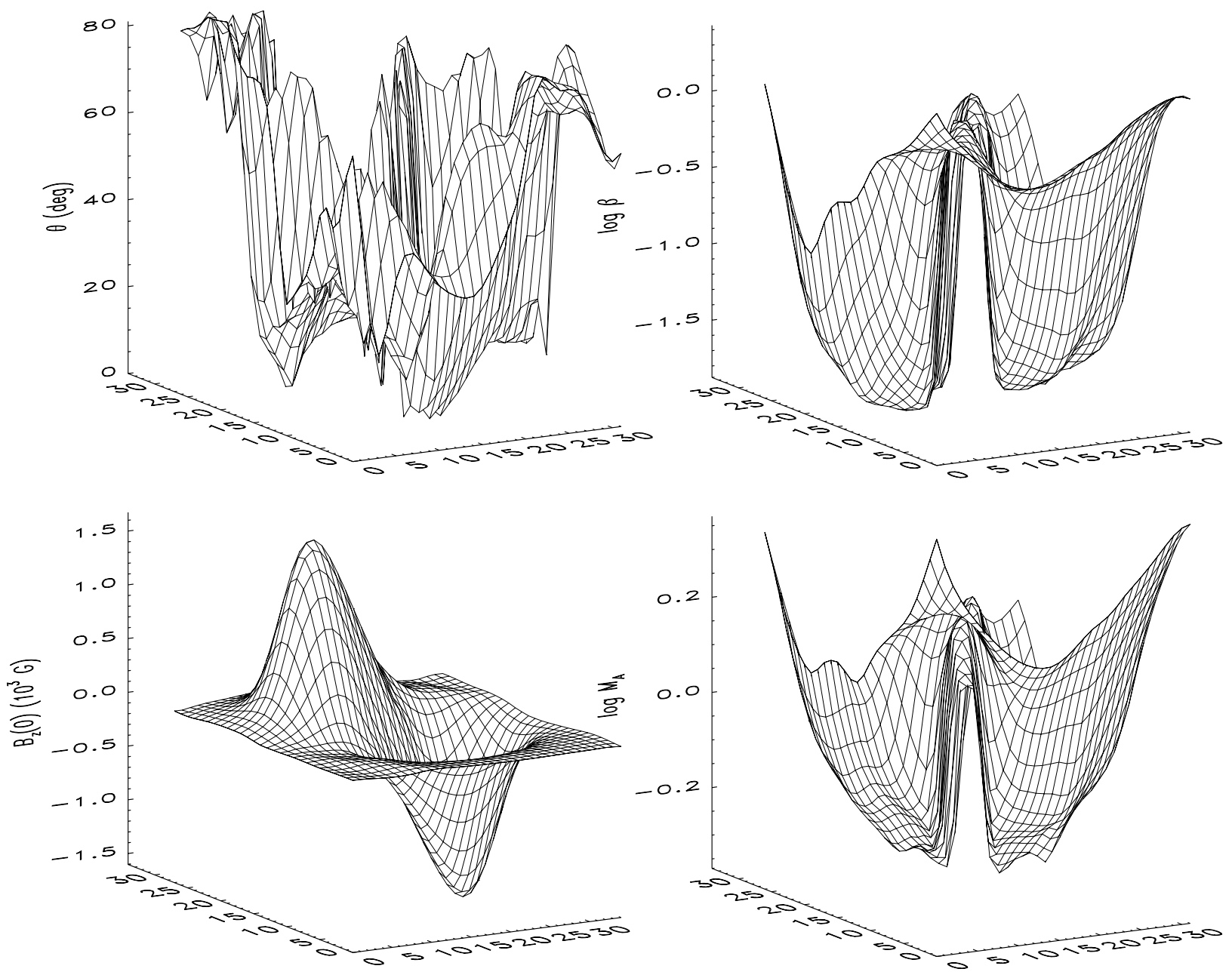

Fig. 5.- The angle $\theta$ (defined in the text) along a slice positioned $8000 \mathrm{~km}$ above the emerging active region (top left frame). Only the portion of the computational domain directly above the emerging structure is shown; the photospheric value of $B_{z}$ (in units of $10^{3}$ $\mathrm{G}$ ) is shown in the lower left frame for reference. $\log \beta$ and $\log M_{A}$ (where $M_{A}$ is the Alfvén Mach number) for this slice are shown in the upper and lower right frames respectively. 
This is an unedited preprint of an article accepted for publication in Astrophysical Journal. The final published article may differ from this preprint.

Copyright 2002 by The American Astronomical Society. Please cite as 'ApJ preprint doi:10.1086/344613'.

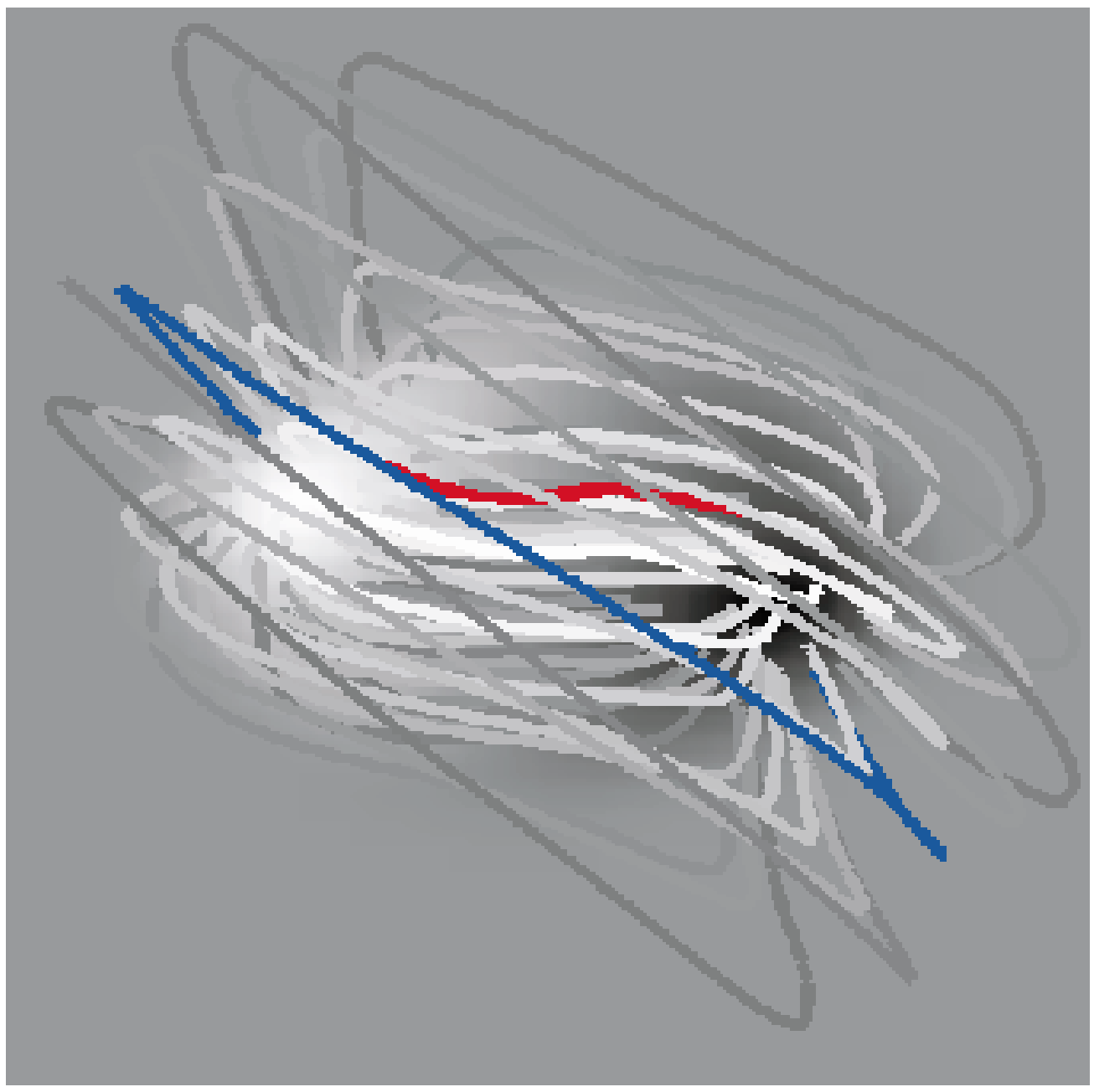

Fig. 6.- A snapshot of the fieldlines emanating from some of the strongest field regions of an emerging bipole. As in Figure 2, the lightest fieldlines are those which emanate from regions where the vertical photospheric magnetic field is the strongest. Two fieldlines are highlighted to show that, depending on which fieldline or which portion of a loop contains emitting plasma, a "sigmoid"-like structure can exhibit either an S-shape or a Z-shape. 
This is an unedited preprint of an article accepted for publication in Astrophysical Journal. The final published article may differ from this preprint.

Copyright 2002 by The American Astronomical Society. Please cite as 'ApJ preprint doi:10.1086/344613'.

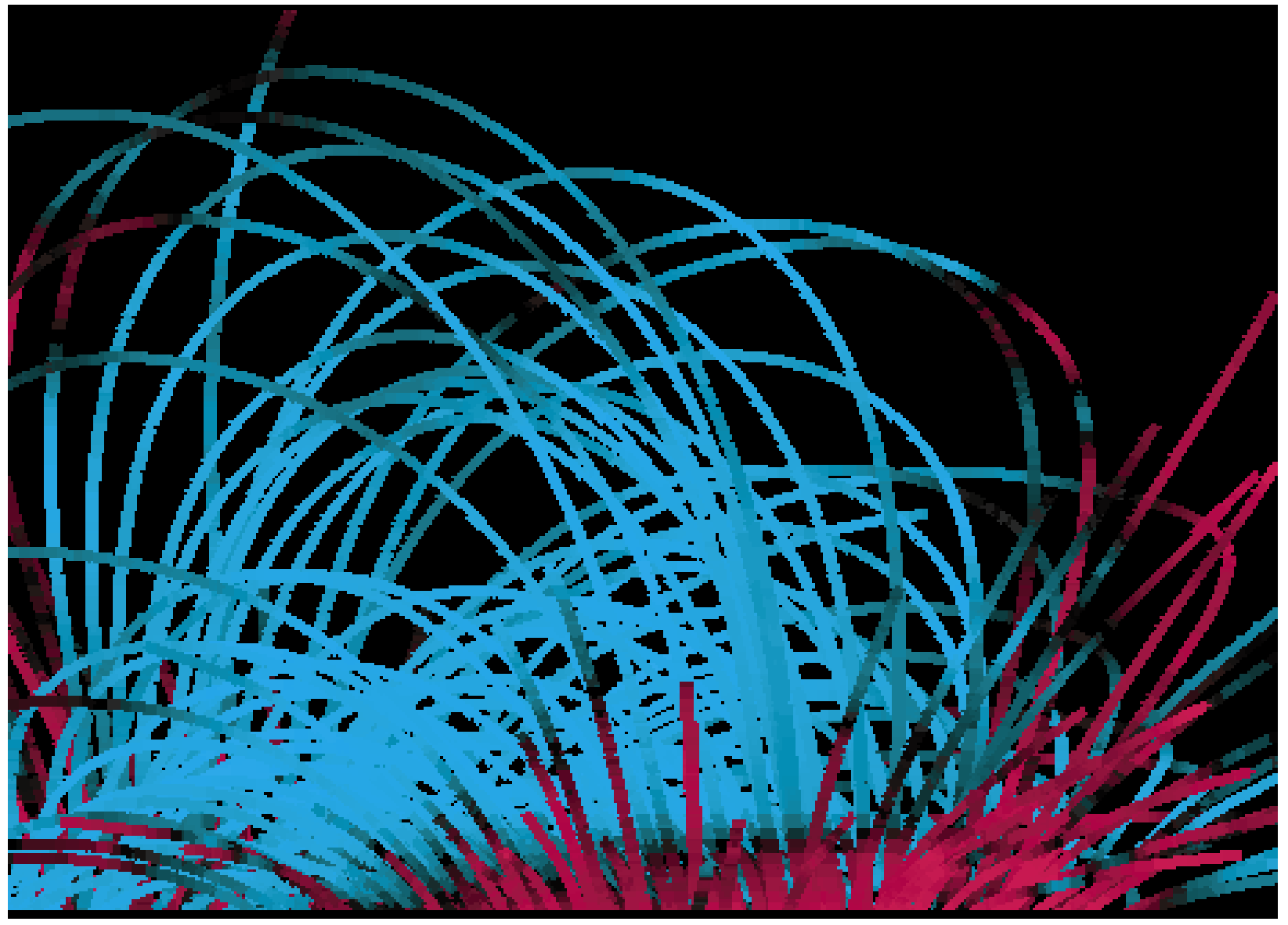

Fig. 7.- A plot of $\theta$ similar to Figure 4 (for the simulation driven by run S3) for a time later in the simulation $(t=9.25)$ when vertical flows have diminished. 